\title{
White matter changes in psychosis risk relate to development and are not impacted by the transition to psychosis
}

\author{
Maria A. Di Biase $\mathbb{B}^{1,2} \cdot$ Suheyla Cetin-Karayumak $\mathbb{1}^{1,3} \cdot$ Amanda E. Lyall $\mathbb{1}^{1,4} \cdot$ Andrew Zalesky $^{2} \cdot$ \\ Kang Ik Kevin Cho ${ }^{1}$. Fan Zhang $\mathbb{D}^{3} \cdot$ Marek Kubicki $\mathbb{B}^{1,3,4} \cdot$ Yogesh Rathi ${ }^{1,3} \cdot$ Monica G. Lyons ${ }^{1} \cdot$ Sylvain Bouix $\mathbb{B}^{1}$. \\ Tashrif Billah $^{1}$ - Alan Anticevic ${ }^{5}$. Charlie Schleifer ${ }^{6} \cdot$ Brendan D. Adkinson $^{7}$. Jie Lisa Ji $\varpi^{5} \cdot$ Zailyn Tamayo $^{5}$.

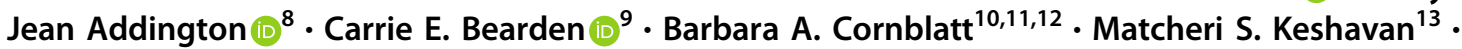 \\ Daniel H. Mathalon ${ }^{14,15}$. Thomas H. McGlashan ${ }^{16} \cdot$ Diana O. Perkins $^{11,17} \cdot$ Kristen S. Cadenhead $^{18}$. \\ Ming T. Tsuang $\mathbb{D}^{18} \cdot$ Scott W. Woods ${ }^{5} \cdot$ William S. Stone ${ }^{13} \cdot$ Martha E. Shenton $^{1,3,4} \cdot$ Tyrone D. Cannon $^{5}$. \\ Ofer Pasternak ${ }^{1,3}$
}

Received: 22 November 2020 / Accepted: 14 April 2021

\begin{abstract}
Subtle alterations in white matter microstructure are observed in youth at clinical high risk (CHR) for psychosis. However, the timing of these changes and their relationships to the emergence of psychosis remain unclear. Here, we track the evolution of white matter abnormalities in a large, longitudinal cohort of CHR individuals comprising the North American Prodrome Longitudinal Study (NAPLS-3). Multi-shell diffusion magnetic resonance imaging data were collected across multiple timepoints (1-5 over 1 year) in 286 subjects (aged 12-32 years): 25 CHR individuals who transitioned to psychosis (CHR-P; 61 scans), 205 CHR subjects with unknown transition outcome after the 1-year follow-up period (CHR-U; 596 scans), and 56 healthy controls (195 scans). Linear mixed effects models were fitted to infer the impact of age and illness-onset on variation in the fractional anisotropy of cellular tissue $\left(\mathrm{FA}_{\mathrm{T}}\right)$ and the volume fraction of extracellular free water (FW). Baseline measures of white matter microstructure did not differentiate between HC, CHR-U and CHR-P individuals. However, age trajectories differed between the three groups in line with a developmental effect: CHR-P and CHR-U groups displayed higher $\mathrm{FA}_{\mathrm{T}}$ in adolescence, and $4 \%$ lower $\mathrm{FA}_{\mathrm{T}}$ by 30 years of age compared to controls. Furthermore, older CHR-P subjects $(20+$ years) displayed $4 \%$ higher FW in the forceps major $(p<0.05)$. Prospective analysis in CHR-P did not reveal a significant impact of illness onset on regional $\mathrm{FA}_{\mathrm{T}}$ or $\mathrm{FW}$, suggesting that transition to psychosis is not marked by dramatic change in white matter microstructure. Instead, clinical high risk for psychosisregardless of transition outcome-is characterized by subtle age-related white matter changes that occur in tandem with development.
\end{abstract}

\section{Introduction}

Studies of individuals at clinical-high risk for developing psychosis (CHR) provide a powerful means to inform on putative mechanisms underlying progression to psychosis. Cumulative evidence suggests that subtle alterations in

Supplementary information The online version contains supplementary material available at https://doi.org/10.1038/s41380021-01128-8.

Maria A. Di Biase

dibiasem@unimelb.edu.au

Extended author information available on the last page of the article white matter microstructure emerge prior to the onset of frank psychosis, which may suggest a preexisting neurodevelopmental anomaly [1]. However, the timing of these changes and their relationships to the emergence of psychosis remain unclear.

More than 25 diffusion magnetic resonance imaging (dMRI) studies have examined white matter microstructure within a prodromal risk paradigm (Supplementary Table 1). Most studies observe cross-sectional lower fractional anisotropy (FA) estimated from diffusion tensor imaging (DTI) in CHR subjects compared to healthy controls [2-12], particularly in the corpus callosum, superior and inferior longitudinal fasciculi, inferior fronto-occipital fasciculi (IFOF), uncinate fasciculi (UF) and cingulum bundle. 
However, several studies have failed to replicate these differences [13-15] or, paradoxically, have observed higher FA in CHR subjects compared to controls within the same white matter fiber bundles $[6,11,16]$. Mixed findings are perhaps not surprising given the substantial heterogeneity of CHR populations with respect to clinical profiles and outcomes [17].

Prospective studies of individuals before the emergence of psychosis raise the important question of timing and course of white matter changes in relation to psychosis onset [18]. Longitudinal studies that track gray matter volume and thickness have revealed rapid declines in the first 2 years following transition to psychosis, which then plateau thereafter [19-27]. These findings mark psychosis onset as a neurobiologically dynamic event, associated with changes in cortical gray matter. However, it is unclear whether white matter alterations follow the same course, characterized by a pinnacle moment co-occurring with transition to psychosis. Three (out of four) previous dMRI studies that separately examined CHR subjects who transitioned to psychosis (CHR-P) detected baseline alterations in these individuals, notably prior to psychosis onset $[9,28,29]$. Therefore, a departure from healthy white matter may coincide with other illness/medication or developmental (age-related) factors.

It is essential to consider variability in white matter pathology against the backdrop of substantial brain reorganization and white matter development during adolescence and early adulthood [30, 31]. This point is further reinforced by cumulative genetic and molecular evidence that implicates early developmental biology in the white matter disruptions associated with established psychosis $[32,33]$. Notably, age-related white matter changes (i.e., in measures of white matter microstructure) are frequently reported in cross-sectional studies of individuals with established illness [34-36]. However, it is not known whether transition to psychosis marks a tipping point for white matter decline and/or whether these changes reflect a gradually evolving developmental process. Testing these hypotheses requires prospective study designs in people who may be at imminent risk of psychosis, which is challenging in terms of recruitment and data collection. As such, the small to modest samples-fewer than 50 CHR subjects and fewer than 10 CHR-P subjects-utilized in previous dMRI studies may have precluded detailed characterization of white matter with respect to its course and timing of alteration.

Here, we examined cross-sectional and longitudinal white matter maturation in the largest diffusion-based imaging population of CHR individuals to date, obtained from the first half sample of the third iteration of the North American Prodrome Longitudinal Study (NAPLS-3). We aimed to examine the evolution of white matter alterations in groups of CHR-P and CHR individuals with unknown transition outcomes (CHR-U) after the study period (1year), to establish whether changes coincide with psychosis onset and/or with maturational processes. A multi-shell dMRI acquisition and between-site harmonization protocols were used to derive measures of microstructure comprising distinct white matter compartments. These included tissuerelated white matter anisotropy $\left(\mathrm{FA}_{\mathrm{T}}\right)$, capturing diffusion in the vicinity of tissue, extracellular free water $(\mathrm{FW})$, reflecting a putative index of unrestricted extracellular water content and FA, reflecting a non-specific index of white matter microstructure that is commonly used in dMRI studies [37]. Linear mixed effects (LME) models were fitted to infer developmental trajectories and temporal changes centered around transition in order to infer the impact of psychosis onset on white matter microstructure. A secondary analysis screened for associations between white matter profiles and a range of neurobehavioral phenotypes in order to determine whether or not white matter changes relate to premorbid/developmental risk factors and/or to current clinical profiles (e.g., severity of positive and negative symptoms).

\section{Methods}

\section{Participants}

This study was approved by local Institutional Review Board committees and informed consent was obtained from all study participants. This study utilized neuroimaging and clinical data collected across eight sites (Supplementary Table 2.1) and at multiple (i.e., 1-5) timepoints (at $\sim 2$ month intervals collected over a 12-month period) in 286 subjects (age range $=12-32$ years). Subjects included 25 CHR individuals who transitioned to psychosis (CHR-P) during the follow-up period (described below), 205 CHR subjects whose long-term transition status was unknown (CHR-U) and who did not transition to psychosis within the study period, and 56 healthy control (HC) subjects who were age and sex-matched to the two CHR groups. This study includes subjects from the first-half sample of NAPLS-3, which is an ongoing prospective analysis of CHR individuals. The overall methodology of NAPLS-3 has been described in detail elsewhere [38]. This current analysis included subjects with available dMRI data.

CHR subjects were referred by health care providers, educators, or social service agencies, or they self-referred in response to program websites and community education efforts. Potential participants underwent a telephone screen and those who screened positive were invited to an inperson eligibility and consent evaluation across eight North American sites. Individuals were interviewed to confirm 
CHR status according to the Criteria of Psychosis-Risk Syndromes (COPS), which is based on the Structured Interview for Psychosis-Risk Syndromes (SIPS) [39]. Briefly, CHR patients met criteria for one or more of the following categories:

1. Sub-threshold intensity/frequency of positive symptoms.

2. First-degree relative with a history of psychosis or has a schizotypal personality disorder accompanied by a decline in general functioning.

3. Brief limited intermittent psychotic symptoms with spontaneous remission within 1 week.

A total of $25 \mathrm{CHR}$ participants transitioned to psychosis during the follow-up period as determined by meeting the SIPS Presence of Psychotic Symptoms (POPS) [40] criteria. Transition criteria require that at least one of the five SIPS positive symptom scales reach a psychotic level of intensity (rated 6) for a frequency of $\geq 1$ hour per day for 4 days per week during the past month or that symptoms seriously impacted functioning (e.g., severely disorganized and/or dangerous to self or others).

Exclusion criteria for all subjects included a current or lifetime Axis I psychotic disorder (including affective psychosis) as measured by the Structured Clinical Interview for DSM-IV Axis I Disorders (SCID) [41], IQ $<70$, a history of a central nervous system disorder, or substance dependence in the past 6 months.

\section{dMRI acquisition}

All participants underwent MRI scanning across eight NAPLS sites on 11 different scanners (Supplementary Tables 2.1 and 2.2), with the same acquisition protocol. Human and phantom data across all sites were compared to rule out gross site effects. Multi-shell dMRI was acquired with 30 gradient directions at $b=1000 \mathrm{~s} / \mathrm{mm}$ [2], six at $b=$ $500 \mathrm{~s} / \mathrm{mm} 2$, three at $b=200 \mathrm{~s} / \mathrm{mm} 2$, and six interleaved b0 images. An additional high b-value shell was acquired, but not used here. The sequence included 70 contiguous axial slices, a $256 \mathrm{~mm}$ field of view, $2 \mathrm{~mm}$ isotropic voxels, a repetition time of $10200 \mathrm{~ms}$, and an echo time of $106 \mathrm{~ms}$.

\section{Harmonization}

To mitigate scanner differences that can induce nonlinear variation in brain maps, a robust retrospective harmonization procedure was applied to the raw dMRI data across the 11 different scanners (Supplementary Table 2.2) [34, 42]. In brief, this involved selecting a target scanner and computing a nonlinear transformation from each of the remaining 10 scanners to the target scanner, based on robust features derived from decomposing the dMRI data into a common mathematical basis using spherical harmonics. Importantly, this procedure removes voxel-level nonlinear discrepancies across scanners on the raw data itself, which then allows the fitting of dMRI models to harmonized data. Applying harmonization to the raw data also circumvents the application of post-hoc statistical methods, such as including scanner as a covariate in a regression model, which is linear and less robust. Harmonization was performed separately for each bvalue shell by selecting a reference scanner (scanner 11, see Supplementary Fig. 1) and mapping dMRI data from the remaining scanners to this reference. A subset of controls were selected from each scanner, age and sex-matched to the reference control subset as targets for harmonization. This method successfully removed scanner-specific effects, such that statistically significant differences $(p<0.01)$ in whole-brain FA between the matched controls across scanners before harmonization were no longer significant after harmonization (Supplementary Fig. $1 ; p>0.05$ ).

\section{Image processing}

\section{Preprocessing}

dMRI data were pre-processed with FMRIB Software Library (FSL) [43], during which 76 scans were removed

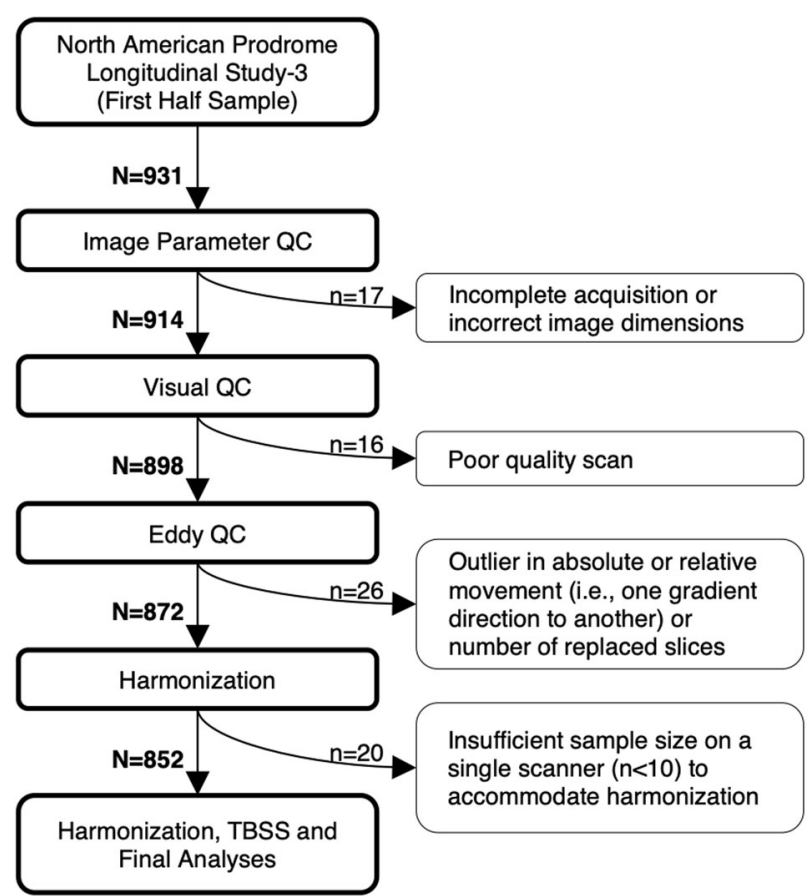

Fig. 1 Flowchart depicting the selection of suitable scans. Each scan underwent several quality control (QC) procedures, followed by harmonization to mitigate scanner/site differences. The flowchart displays the number of discarded and remaining scans following each process. 
before arriving at the final scan number of 852 (Fig. 1), comprising 286 subjects in total (CHR-P $=25$; CHR-U = $205 ; \mathrm{HC}=56$ ). Each scan was checked for correct image parameters and visually inspected for poor image quality. Remaining scans were corrected for motion and gradientinduced eddy currents using FSL's Eddy [44, 45]. Eddy outputs were used to detect dMRI image outliers ( $>3$ SDs above the mean) on absolute movement, relative movement or number of outlier slices replaced with Gaussian function.

\section{dMRI metric computation and TBSS}

FA volumes were computed by fitting a single-diffusion tensor to the dMRI data and FW and $\mathrm{FA}_{\mathrm{T}}$ volumes were computed by free-water imaging, which applies a regularization framework to fit a two compartment model to the dMRI data [37]. The two-compartment model separates the contribution of free water from water molecules diffusing in the vicinity of tissue. The fractional volume of FW requires large enough spaces that constitute unrestricted extracellular water molecules around and between myelin and axolemma. An in-house protocol was used (https://github.com/pnlbwh/TBSS), which nonlinearly registers FA maps to the ENIGMA-DTI target [46] using Advanced Normalization Tools (ANTs) [47]. The individually registered brains were projected onto the ENIGMA-DTI white matter skeleton with FSL's TBSS [48], which was employed to alleviate any residual misalignment error. The FA-derived transformations and projection parameters were used to project $\mathrm{FW}$ and $\mathrm{FA}_{\mathrm{T}}$ maps onto the skeleton mask. Statistical inference was performed on the resulting skeletonized maps ( $\mathrm{FW}, \mathrm{FA}_{\mathrm{T}}$ and $\left.\mathrm{FA}\right)$.

For each subject, a total of 14 fibers-of-interest (FOI) were extracted from the Illinois Institute of Technology (IIT) Human Brain probabilistic atlas version 4.1, using a threshold of 0.25 . FOIs were selected based on commonly implicated regions in previous CHR studies published between 2008 and 2019 (Supplementary Table 1). For each FOI, one value was extracted by averaging each dMRI measure (i.e., $\mathrm{FA}, \mathrm{FW}, \mathrm{FA}_{\mathrm{T}}$ ) respectively across all voxels traversing a probabilistic fiber bundle. To increase power and to reduce the total number of variables, intrahemispheric fibers were averaged across hemispheres to yield a total of 8 regions-of-interest (ROIs), including 7 FOIs and whole-brain/average skeletonized white matter.

\section{Neurobehavioral phenotypes}

Supplementary Table 3 displays clinical assessment scales that were administered at baseline and some again at followup (denoted by asterisks). A total of 49 baseline (Supplementary Table 4) and 11 longitudinal (Supplementary Table 5) variables were examined. Some neurobehavioral variables were re-scaled so that higher scores indicate worse performance/higher symptom severity. Missing data were handled with an iterative procedure: first, subjects with $>20 \%$ of missing elements were removed (3 subjects) and second, the remaining missing elements were imputed using probabilistic principal component analysis (PPCA; Supplementary Tables 4 and 5) [49].

\section{Statistical modeling}

\section{Demographics and timepoint characteristics}

One-way Analysis of variance (ANOVA) and Chi-square tests evaluated differences in age and sex proportions between the three study groups (CHR-P, CHR-U, and HC) at baseline and across timepoints, as well as the number of timepoints and interscan intervals between the study groups.

\section{Between-group differences in white matter microstructure}

Between-group comparisons in the white matter dMRI parameters ( $\mathrm{FW}, \mathrm{FA}_{\mathrm{T}}$, and $\mathrm{FA}$ ) were performed using FSL's Randomise for voxel-wise analyses [50], and Matlab for ROI analyses. Baseline differences (286 scans) were examined with a general linear model (GLM) to assess the null hypothesis of equality in dMRI measures between CHR-U and HC, and between CHR-P and HC, while controlling for the potential confounding effects of age and sex. Multiple comparisons correction was performed with threshold-free cluster enhancement [51] for the voxel-wise analyses and with the false discovery rate (FDR) for regional analyses (8 ROIs).

\section{Modeling the impact of age and psychosis onset on white matter microstructure}

Linear mixed effects (LME) models were fitted to infer developmental trajectories of FA (shown in Supplementary Material), $\mathrm{FA}_{\mathrm{T}}$ and $\mathrm{FW}$ across age for CHR-P, CHR-U and $\mathrm{HC}$ groups (fixed effects), while accounting for repeated measurements (random effects; refer to Supplementary Material for details). LME models were independently fitted with age centered between 12 and 32 years in yearly increments; that is, $\mathrm{A}=12,13, \ldots 32$. When the LME was estimated with age centered at $\mathrm{A}$, the main effects of group $\left(\beta_{2}\right.$ and $\beta_{3}$ ) specifically pertained to group differences at the age of A years. Therefore, fitting the model independently at each year yielded cross-sectional snapshots of dMRI indices across the entire age range of CHR-P, CHR-U and $\mathrm{HC}$ subjects [35].

LME models were further fitted in the CHR-P population ( $n=25$ with a total of 61 scans) to investigate the impact of psychosis onset (operationalized as scan date minus psychosis onset date) on variation in dMRI measures, while 
Table 1 Sample and timepoint characteristics.

\begin{tabular}{llllll}
\hline & HC & CHR-U & CHR-P & $f$ & $p$ \\
& Mean (SD or \%) & Mean (SD or \%) & Mean (SD or \%) & & \\
\hline Baseline age & $20.65(5.09)$ & $19.20(4.09)$ & $19.58(4.02)$ & 2.54 & $>0.05$ \\
Average age (across timepoints) & $20.91(5.09)$ & $19.41(4.11)$ & $19.72(4.10)$ & 2.67 & $>0.05$ \\
Sex (males/females) & $27 / 29(48 \%)$ & $104 / 101(51 \%)$ & $13 / 12(52 \%)$ & 0.14 & $>0.05$ \\
Number of timepoints ${ }^{\mathrm{a}}$ & $3.48(1.21)$ & $2.91(1.53)$ & $2.44(1.12)$ & 5.44 & 0.005 \\
Interscan Interval (months) & $11.36(2.73)$ & $8.43(5.50)$ & $9.60(4.90)$ & 1.58 & $>0.05$ \\
Number with longitudinal data & $53(95 \%)$ & $144(70 \%)$ & $20(80 \%)$ & & \\
Scans at each timepoint & $n$ & $n$ & $n$ & & \\
$1(n=286)$ & 56 & 205 & 25 & & \\
$2(n=217)$ & 53 & 144 & 11 & & \\
$3(n=177)$ & 44 & 122 & 3 & & \\
$4(n=112)$ & 27 & 82 & 2 & & \\
$5(n=60)$ & 15 & 596 & 61 & & \\
TOTAL SCANS $(n=852)$ & 195 & & & & \\
\hline
\end{tabular}

${ }^{a}$ Denotes a significant between-group difference. accounting for age and sex, as well as the random betweensubject variability. Therefore, the impact of psychosis onset was examined in a strictly prospective (i.e., within subjects) manner.

\section{Relationships between white matter microstructure and neurobehavioral phenotypes}

Canonical correlation analysis (CCA) was used to examine multivariate associations between sets of neurobehavioral measures and sets of dMRI measures, which is more principled than independently testing each potential association and correcting for multiple comparisons across the neurobehavioral and dMRI measures [52]. Two CCA's were performed to examine associations at baseline (baseline $\mathrm{dMRI}$ and baseline neurobehavioral phenotypes) and in changes across time $(\Delta \mathrm{dMRI}$ phenotypes and $\Delta$ neurobehavioral phenotypes). Changes in dMRI and neurobehavioral phenotypes were quantified by their slope, estimated using linear regression across all available timepoints for a given subject. CCA quantified modes of covariation by identifying linear compositions that maximize correlation between the composite neurobehavioral score and the composite dMRI score. Data included matrices for neurobehavioral phenotypes (baseline: $227 \times 49$; longitudinal: $167 \times 11$ ) and dMRI data (baseline: $227 \times 24$; longitudinal: $167 \times 24)$. Baseline neurobehavioral and dMRI matrices were adjusted for the potential confounding effects of age, sex and site (site confound relates only to the neurobehavioral data). Change in neurobehavioral and dMRI matrices were further adjusted for time-related confounds including the number of timepoints available for each subject, mean age across timepoints and age differences between the first and last timepoint. To avoid overfitting, dimensionality reduction with principal component analysis (PCA) was applied to symptom matrices, retaining a set of principal components that captured the most variance, as described in Taquet et al. [53]. The resulting data-reduced neurobehavioral and dMRI matrices were inputs to the CCA. Permutation testing was used to evaluate statistical significance.

\section{Results}

\section{Demographics}

Demographics and timepoint characteristics are shown in Table 1. A total of 25 individuals (12.2\% of all CHR individuals) met POPS criteria for transition to psychosis and were thus categorized as CHR-P (mean transition age, mean $[\mathrm{SD}]=19.73$ [4.42]; mean time of transition since baseline, mean $[\mathrm{SD}]=4.8$ months [6.39]). Diagnostic outcomes for the CHR-P individuals were as follows: unspecified schizophrenia ( $n=11,44 \%$ ), unspecified psychosis ( $n=4,16 \%)$, schizoaffective disorder $(n=4,16 \%)$, schizophreniform $(n=3,12 \%)$, primary mood disorder with secondary psychosis $(n=2,8 \%)$, missing diagnostic outcome $(n=1,4 \%)$.

There was no significant difference in sex or age (at baseline or across timepoints) between the three study groups (CHR-P, CHR-U, and HC). While there was no significant difference in the interscan interval between the study groups, HC completed significantly more follow-up assessments compared to the CHR-P and CHR-U groups. 


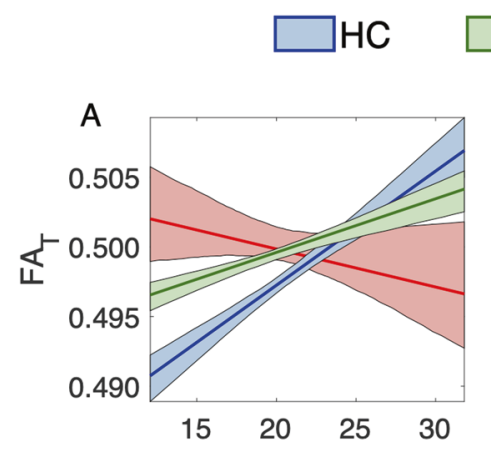

$\square$ CHR-U $\square$ CHR-P
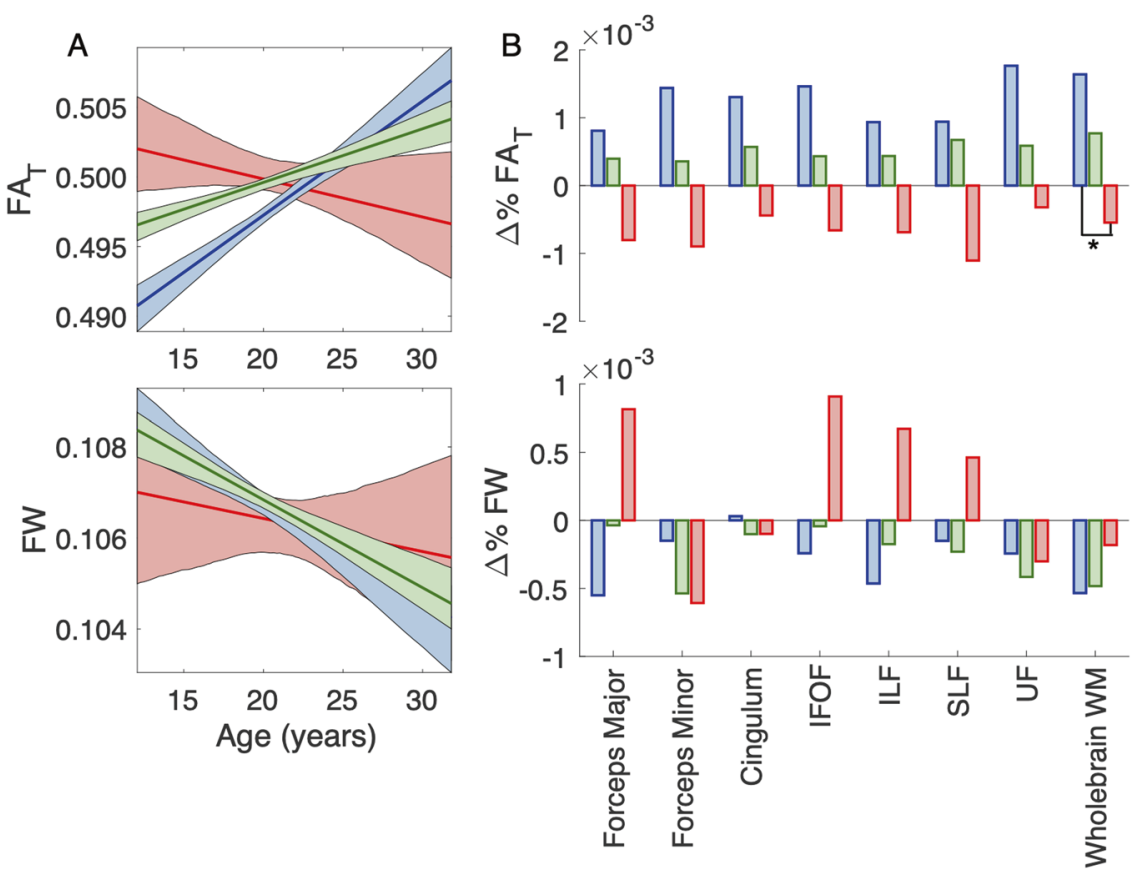

Fig. 2 Age-related change in white matter microstructure. A presents curves modeling age-related change of white matter tissue anisotropy $\left(\mathrm{FA}_{\mathrm{T}}\right)$ and free water $(\mathrm{FW})$ in healthy controls $(\mathrm{HC}$; blue curve; $\beta_{1}+\beta_{4} \times$ Age), clinical high-risk individuals with unknown transition outcomes (CHR-U; green curve; $\beta_{1}+\beta_{2}+\left(\beta_{4}+\beta_{5}\right) \times$ Age) and in CHR individuals who transitioned to psychosis (CHR-P; red curve; $\beta_{1}+\beta_{2}+\left(\beta_{4}+\beta_{6}\right)+$ Age). Shaded areas reflect $95 \%$ confidence intervals estimated with bootstrapping (1000 samples). B

\section{Between-group differences}

No overall between-group differences were seen for FA, $\mathrm{FA}_{\mathrm{T}}$ or $\mathrm{FW}$, measured on a voxel-wise or regional basis after FDR correction (see Supplementary Tables 6-8 for descriptives and statistics). Therefore, baseline measures of white matter microstructure did not differentiate between HC, CHR-U, or CHR-P.

\section{The impact of age on white matter microstructure}

Figure 2 presents age trajectories for whole-brain $\mathrm{FA}_{\mathrm{T}}$ and FW and rates of change for the 8 ROIs (see Supplementary Tables 9-11 for age statistics across ROIs). The rate of change in whole-brain $\mathrm{FA}_{\mathrm{T}}$ differed between CHR-P and $\mathrm{HC}$, which survived FDR correction $\left(\beta_{6}=-0.129 ; t=\right.$ -2.6 ; FDR $p=0.009$ ). This result remained significant after controlling for a range of medication factors (see Supplementary Table 12). CHR-U subjects displayed trajectories characterized by intermediate $\mathrm{FA}_{T}$ values between HC and CHR-P. Throughout adolescence and early adulthood, both CHR-P and CHR-U groups displayed higher $\mathrm{FA}_{\mathrm{T}}$ compared to $\mathrm{HC}$ in whole-brain white matter (Fig. 3). presents the percent of age-related change per year (i.e., slope) in FA, $\mathrm{FA}_{\mathrm{T}}$ and FW averaged across each of the 8 ROIs, in HC $\left(\beta_{4}\right)$, CHR-U $\left(\beta_{4}+\beta_{5}\right)$ and CHR-P $\left(\beta_{4}+\beta_{6}\right)$ groups. The $\mathrm{FA}_{\mathrm{T}}$ slope in whole-brain white matter significantly differed between CHR-P and HC (FDR $p<$ 0.05 , as indicated by the asterisk). Negative percentages indicate agerelated decline in dMRI values. IFOF inferior fronto-occipital fasciculus, ILF inferior longitudinal fasciculus, SLF superior longitudinal fasciculus, UF uncinate fasciculus, WM white matter.

However, by 30 years of age, $\mathrm{FA}_{\mathrm{T}}$ in CHR-P and CHR-U fell below that of $\mathrm{HC}$, with regionally significant reductions observed in the forceps minor and superior longitudinal fasciculus among CHR-P subjects $(p<0.05$; Fig. 3). With regard to $\mathrm{FW}$, there were no significant between-group differences in the rates of change in whole-brain white matter for CHR-U $\left(\beta_{5}=0.0278 ; t=1.0 p>0.05\right)$ or for CHR-P $\left(\beta_{6}=0.0667 ; t=1.33 ; p>0.05\right)$ compared to HC. However, significantly higher FW was seen in older $(20+$ years of age) CHR-P subjects compared to $\mathrm{HC}$ within the forceps major $(p<0.05$; Fig. 3$)$.

\section{The impact of psychosis onset on white matter microstructure}

The impact of psychosis onset (i.e., scan date minus psychosis onset date) in CHR-P subjects was not significant across regional FA, $\mathrm{FA}_{\mathrm{T}}$ or $\mathrm{FW}$ measures $\left(\beta_{2}, \mathrm{FDR} p>0.05\right.$; see Supplementary Table 13 for statistics). Therefore, transition to psychosis did not predict variation in white matter microstructure. 

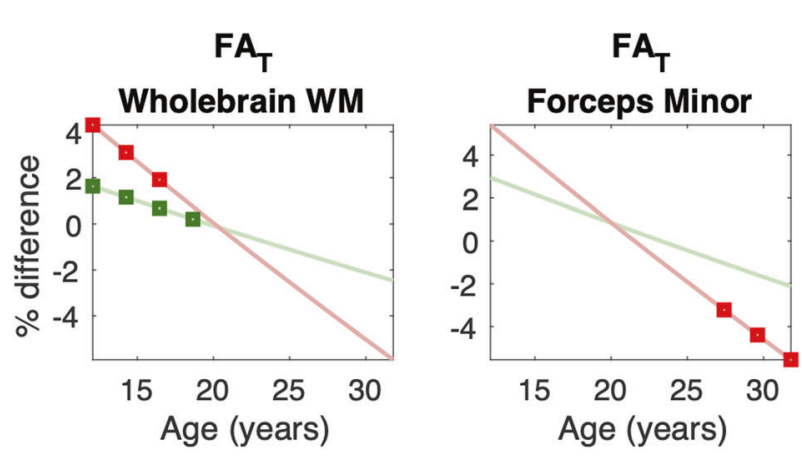

Fig. 3 Significant group differences across ages in white matter microstructure. Graphs present between-group differences in tissue $\mathrm{FA}\left(\mathrm{FA}_{\mathrm{T}}\right)$ and free water $(\mathrm{FW})$ as a function of age, quantified by $\beta_{3} /$ $\beta_{1} \times 100 \%$ in clinical high-risk with unknown transition outcomes (CHR-U) and by $\beta_{4} / \beta_{1} \times 100 \%$ in CHR individuals who transitioned to psychosis (CHR-P). Negative percent $(\%)$ differences indicate lower values in CHR-U and CHR-P relative to healthy comparison (HC)

\section{Relationships between white matter microstructure and neurobehavioral phenotypes}

Canonical correlation analysis (CCA; Fig. 4A) identified a highly significant mode of neurobehavioral-dMRI covariation, measured at baseline ( $r=0.69$, permutation test: $p<$ $10-5)$. As shown in Fig. 4B, the composite dMRI score significantly covaried with higher severity of psychopathology (positive, negative, disorganized, schizotypal, and depressive symptoms) and cognitive disability, as well as with higher cannabis use and poorer functional performance (general, social and independent living domains) and premorbid adjustment in childhood and adolescence (demarcated with black squares in Fig. 4B). All neurobehavioral phenotypes were positively correlated with the composite dMRI score. At the same time, both FA and $\mathrm{FA}_{\mathrm{T}}$ but not FW measures, were significantly associated with the composite neurobehavioral score. These covariation patterns suggest that lower anisotropy relates to more severe phenotypic abnormalities in neurobehavior, as well as to premorbid risk factors in childhood and adolescence.

In terms of longitudinal change, there was a significant mode of covariation between the composite slope of neurobehavior and the composite slope of dMRI measures ( $r=$ 0.67 , permutation test: $p=0.04$; Supplementary Fig. 2). No specific phenotype contributed significantly to this complex pattern of covariation, suggesting that subtle changes spanning the entire composition of neurobehavioral and dMRI parameters drive longitudinal covariation between these measures. Specifically, upturns in symptom severity (positive, negative and disorganized types), alcohol and drug use, and functional disturbances accompanied composite longitudinal changes in white matter microstructure, involving decreases $\mathrm{FA}$ and $\mathrm{FA}_{\mathrm{T}}$-particularly in the
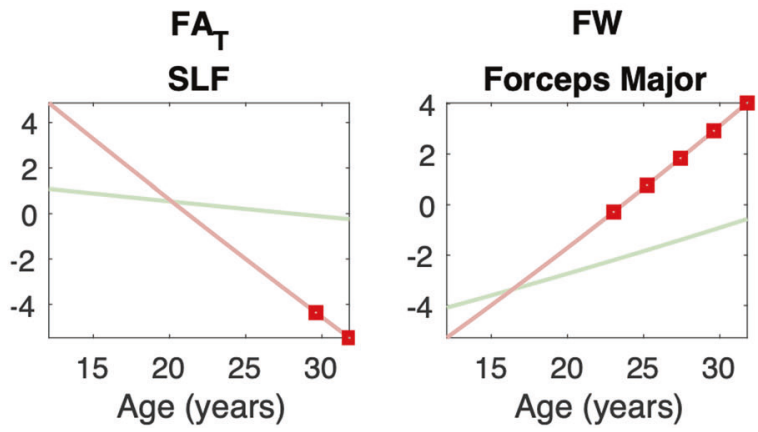

subjects. Average dMRI measures were quantified yearly using age centering between 12 and 32 years. Age epochs at which the average dMRI measure significantly differed between the CHR groups and comparison subjects are denoted with red/green squares (RAWp $<$ 0.05 ), as determined by the significance of the $\beta_{3}$ and $\beta_{4}$ regression coefficients, respectively. SLF superior longitudinal fasciculus.

cingulum and forceps minor-and increases in FW-particularly in the forceps major. These findings collectively suggest that levels of dMRI indices in white matter relate to premorbid risk factors at baseline and covary with features of psychopathology across time.

\section{Discussion}

The major finding here is that individuals classified as CHR display altered age trajectories in white matter microstructure, indexed by $\mathrm{FA}_{\mathrm{T}}$ and FW. Compared to HC, levels of $\mathrm{FA}_{\mathrm{T}}$ were higher in adolescents across both CHR groups, which preceded slower rates of growth in the CHR-U group, and statistically significant decline in the CHR-P group. In contrast, transition to psychosis did not predict variation in white matter microstructure. Regardless of transition outcome, variation in white matter measures covaried with the severity of psychopathology and premorbid risk factors. These results suggest that white matter dMRI measures are sensitive to features of psychopathology and to atypical development trajectories in individuals meeting CHR criteria.

While binary illness outcomes-like transition to psychosis-reflect attractive targets for biological association, the impact of transition time is not reflected in our findings regarding cellular and extracellular white matter among CHR individuals. As such, white matter alterations associated with psychosis risk may not undergo accelerated change around the time of illness onset as compared to changes occurring throughout the prodromal period. However, it remains possible that the rate of white matter change is faster around the time of illness onset compared to that seen in protracted illness or chronic schizophrenia. Notably, 

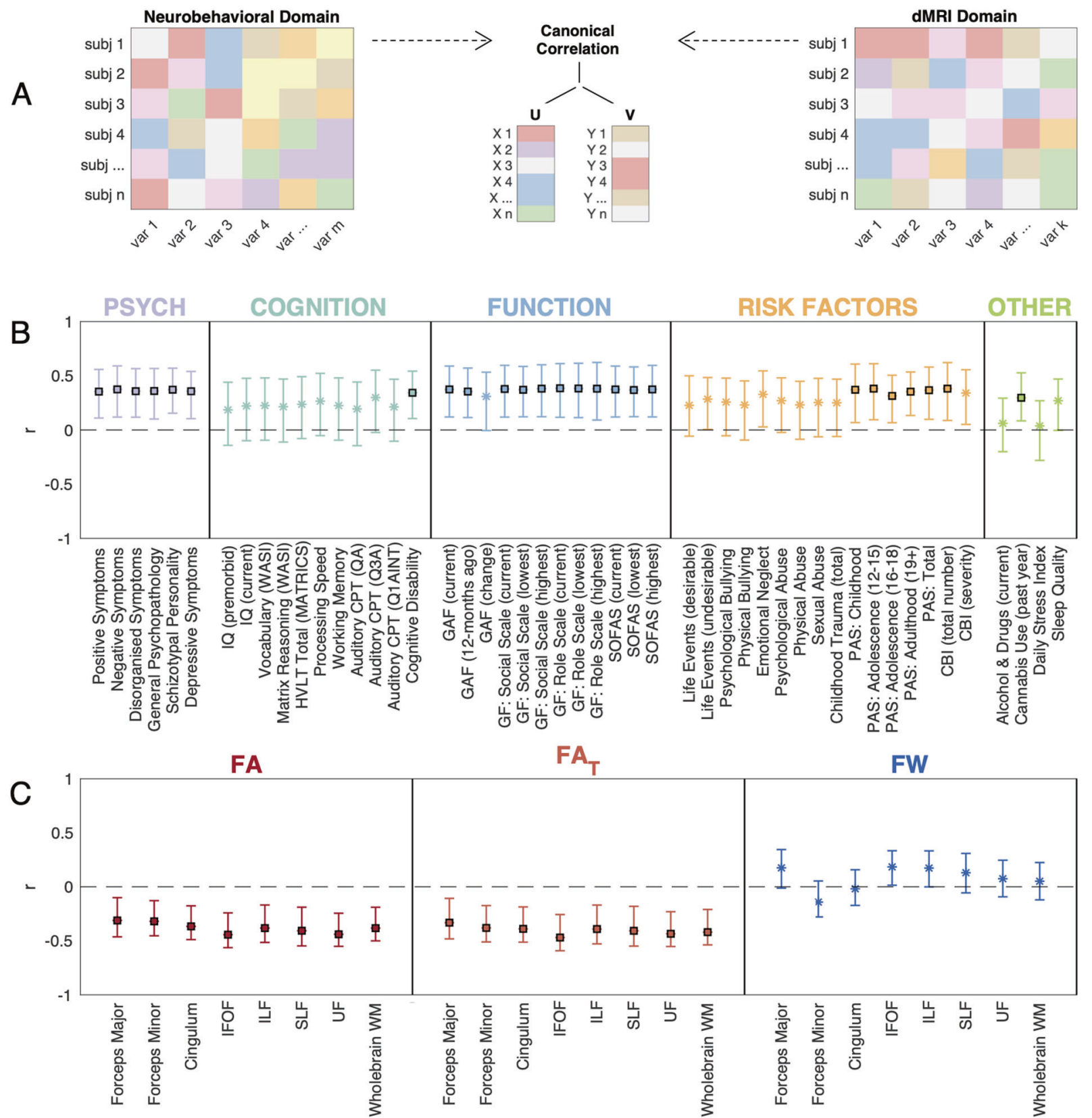

Fig. 4 Association between neurobehavioral and dMRI phenotypes. Panel A presents a schematic of canonical correlation analysis (CCA) to examine multivariate neurobehavior-dMRI covariation at baseline. CCA maximizes covariation between the canonical score for neurobehavioral measures (U), and the canonical score for dMRI measures (V). Panel B presents correlations between each of the 49 neurobehavioural phenotypes with $\mathrm{V}$ and Panel $\mathrm{C}$ presents correlations between each of the $24 \mathrm{dMRI}$ measures with U. Error bars indicate

the time before and/or after psychosis onset monitored in the current study may have been too short to capture a turning point reflected by white matter changes. Furthermore, uncertain outcomes, particularly among individuals lost to follow-up, may have reduced sensitivity to detect overall between-group differences. Future longitudinal
95\% confidence intervals estimated with bootstrapping (1000 samples) and black squares demarcate significant correlations, after FDR correction across the neurobehavioural and dMRI phenotypes respectively. PSYCH psychopathology, IFOF inferior fronto-occipital fasciculus, ILF inferior longitudinal fasciculus, SLF superior longitudinal fasciculus, UF uncinate fasciculus, WM white matter, GAF global assessment of functioning; GF global functioning, PAS premorbid adjustment scale, CBI childhood brain injury.

studies that track longer time periods following psychosis onset are required to evaluate rates of change around the time of illness onset, relative to long-term white matter trajectories.

It is noteworthy, however, that age-related decline in white matter anisotropy has been observed in individuals 
with established schizophrenia-spectrum disorders [34-36], and with non-psychotic disorders, such as bipolar disorder, major depressive disorder and obsessive-compulsive disorder [54-56]. These findings have been interpreted (together with post-mortem findings) as accelerated brain aging, marked by increased myelin breakdown [34-36]. Here, we extend these observations to individuals with sub-threshold psychosis, where our findings suggest that white matter microstructure departs from healthy development prior to diagnosable psychotic illness and in an age-related manner. Moreover, and consistent with prior dMRI studies across the lifespan, healthy controls showed age-related increases in anisotropy (Fig. 2A), coinciding with the known timecourse of myelin development [57]. Both CHR-U and CHR-P groups deviated from this typical developmental course, further suggesting that atypical development may characterize CHR states, regardless of illness outcome, albeit more evidently in those individuals who transition to frank psychosis. Taking these age-related changes into account may help us to appreciate better why conflicting results arise in diffusion studies of CHR populations. Importantly, the transition from higher $\mathrm{FA}_{\mathrm{T}}$ to lower $\mathrm{FA}_{\mathrm{T}}$ (compared with $\mathrm{HC}$ ) manifested gradually at around 20 years of age, explaining the lack of overall group difference (in means) between healthy controls, CHR-U and CHR-P.

Our findings may reflect a shifted trajectory of white matter development in CHR populations, characterized by early maturational peaks and then slower white matter maturation and/or premature white matter decline. Interpreting the cellular basis of shifted white matter trajectories is, however, difficult, due to the inferential nature of diffusion imaging measurements. Nonetheless, our use of freewater imaging eliminates confounders from free water and in turn, implicates cellular tissue changes in abnormal white matter trajectories. In particular, we observed increased $\mathrm{FA}_{\mathrm{T}}$ in whole-brain white matter among younger CHR-P subjects and, to a lesser extent, among CHR-U subjects. This finding provides an important replication of increased white matter anisotropy in CHR, reported in prior diffusion studies $[6,11,16]$. An early peak in $\mathrm{FA}_{\mathrm{T}}$ could possibly signal accelerated myelin development, deficient axonal pruning or oligodendroglial hyperplasia as a compensatory response to white matter defects or to other early/prenatal events.

In addition to higher $\mathrm{FA}_{\mathrm{T}}$ in younger $\mathrm{CHR}$ subjects, older ( $>25$ years) CHR-P subjects displayed regionally reduced $\mathrm{FA}_{\mathrm{T}}$ in the forceps minor and superior longitudinal fasciculi. The forceps minor (anterior part of the corpus callosum) connects homologous prefrontal brain regions, and the superior longitudinal fasciculi represent frontoparietal connections $[58,59]$. Both tracts and their connecting gray matter regions are frequently implicated in the pathophysiology of psychosis risk (see Supplementary Table 1), and facilitate wide-ranging functions, including somatosensory, attention, motor, and language processes [58, 59]. While current dMRI measures are not specific to cellular/molecular mechanisms, localized $\mathrm{FA}_{\mathrm{T}}$ effects may relate to regional variability in white matter structures (e.g., myelin sheath geometry), shaped by a multitude of local cellular events and molecular signaling pathways $[60,61]$. These possibilities could be explored in future multimodal imaging studies that aim to deconstruct potential white matter pathologies in CHR.

When the neurobehavioral phenotypes were viewed in terms of their specific contribution to white matter dMRI profiles, a clear pattern emerged: higher clinical severity at baseline related to lower anisotropy $\left(\mathrm{FA}_{\mathrm{T}}\right.$ and $\left.\mathrm{FA}\right)$ at baseline. These results replicate several previous reports that reduced $\mathrm{FA} / \mathrm{FA}_{\mathrm{T}}$ relates to higher psychopathological severity, cognitive disability, poorer functioning and greater cannabis consumption [11, 62-64], suggesting that dMRI changes co-occur with psychopathology, regardless of specific diagnoses. We also observed a significant effect of poorer premorbid adjustment (e.g., sociability, withdrawal and scholastic performance) in childhood and adolescence on white matter dMRI measures. While the direction of this relationship is unclear (i.e., whether premorbid adjustment reflects a consequence or a precipitant factor in altered white matter development), and may even differ across individuals, it reinforces the importance of early life/ developmental factors in shaping long-term profiles of white matter microstructure.

\section{Limitations and conclusions}

Several methodological considerations related to the present analysis are worthy of note. First, the sample size comprising the CHR-P group in this study is small relative to the CHR-U and HC groups, leading to wide confidence intervals around the white matter trajectories estimated for CHR$\mathrm{P}$, and reduced power to detect changes associated with illness onset. We will examine replicability of our findings in the second half of the NAPLS-3 sample when these data are available. Second, the cellular and molecular basis of dMRI measures remain only partially understood and may vary across CHR individuals. Thus, cellular interpretations remain speculative until requisite large-scale multimodal imaging studies clarify the precise nature of microstructural changes in cellular and extracellular white matter among CHR individuals. Third, it is possible that individuals transitioned to psychosis after the follow-up period and hence, anomalies observed in the CHR-U group are confounded by the potential of illness onset in a subset of the individuals. Alternatively, illness trajectories among the CHR-U individuals may follow a heterotypic course (i.e., attenuated psychotic symptoms that evolve into other types/ 
diagnostic categories) [65], although recent findings call the frequency of heterotypic courses into question [66-68].

Transition to psychosis may not represent a flash point marked by dramatic change in white matter microstructure. Instead, psychopathology associated with CHR states is reflected in subtle white matter changes that occur in tandem with the pace of development. These changes are more pronounced in those who transition to psychosis and are marked by initially higher and later lower diffusion anisotropy in white matter compared to controls. Importantly, variation in white matter microstructure relates not only to the severity of psychopathology, but also to historical premorbid risk factors in childhood and adolescence, which may synergistically alter the path of white matter development. Our findings emphasize the need for holistic clinical approaches that target multiple dimensions of health during the course of development.

\section{Code availability}

The Matlab function fitlme was used to perform linear mixed effects (LME) models with bootstrapping confidence intervals. This code is publicly accessible.

Acknowledgements MADB was supported by an Australian National Health and Medical Research Council (NHMRC) Investigator Grant (1175754). This study was supported by a National Alliance for Research on Schizophrenia \& Depression (NARSAD) Brain and Behavior Research Foundation Young Investigator Award (to AEL) and by the National Institute of Mental Health (grant K01 MH115247-01A1 to AEL; grants R01MH108574, R01MH102377, R01MH074794, P41EB015902 to OP; grant U01MH081984 to JA; grant U01MH081928 to WSS; grant U01MH081944 to KSC; grant U01MH081902 to TDC and CEB; grant U01MH082004 to DOP; grant U01MH082022 to SWW; grant U01MH076989 to DHM; grant U01MH081857 to BAC; and, grant U01MH109977 to MES).

\section{Compliance with ethical standards}

Conflict of interest The authors declare no competing interests.

Publisher's note Springer Nature remains neutral with regard to jurisdictional claims in published maps and institutional affiliations.

\section{References}

1. Di Biase MA, Pantelis C, Zalesky A White Matter Pathology in Schizophrenia. Neuroimaging in Schizophrenia. Springer2020, pp 71-91.

2. Saito J, Hori M, Nemoto T, Katagiri N, Shimoji K, Ito S, et al. Longitudinal study examining abnormal white matter integrity using a tract-specific analysis in individuals with a high risk for psychosis. Psychiatry Clin Neurosci. 2017;71:530-541.

3. Tomyshev AS, Lebedeva IS, Akhadov TA, Omelchenko MA, Rumyantsev AO, Kaleda VG. Alterations in white matter microstructure and cortical thickness in individuals at ultra-high risk of psychosis: A multimodal tractography and surface-based morphometry study. Psychiatry Res: Neuroimaging. 2019;289:26-36.
4. Peters B, Schmitz N, Dingemans P, Van Amelsvoort T, Linszen D, De Haan L, et al. Preliminary evidence for reduced frontal white matter integrity in subjects at ultra-high-risk for psychosis. Schizophrenia Res. 2009;111:192-193.

5. Cho KIK, Shenton ME, Kubicki M, Jung WH, Lee TY, Yun J-Y, et al. Altered thalamo-cortical white matter connectivity: Probabilistic tractography study in clinical-high risk for psychosis and first-episode psychosis. Schizophrenia Bull. 2016;42:723-731.

6. Karlsgodt KH, Niendam TA, Bearden CE, Cannon TD. White matter integrity and prediction of social and role functioning in subjects at ultra-high risk for psychosis. Biol psychiatry. 2009;66:562-569.

7. Wang C, Ji F, Hong Z, Poh J, Krishnan R, Lee J, et al. Disrupted salience network functional connectivity and white-matter microstructure in persons at risk for psychosis: findings from the LYRIKS study. Psychological Med. 2016;46:2771-2783.

8. Epstein KA, Cullen KR, Mueller BA, Robinson P, Lee S, Kumra $\mathrm{S}$. White matter abnormalities and cognitive impairment in earlyonset schizophrenia-spectrum disorders. J Am Acad Child Adolesc Psychiatry. 2014;53:362-372. e361-362

9. Katagiri N, Pantelis C, Nemoto T, Zalesky A, Hori M, Shimoji K, et al. A longitudinal study investigating sub-threshold symptoms and white matter changes in individuals with an 'at risk mental state'(ARMS). Schizophrenia Res. 2015;162:7-13.

10. Fitzsimmons J, Rosa P, Sydnor VJ, Reid BE, Makris N, Goldstein $\mathrm{JM}$, et al. Cingulum bundle abnormalities and risk for schizophrenia. Schizophrenia Res. 2020;215:385-391.

11. Krakauer K, Nordentoft M, Glenth $\varnothing \mathbf{j}$ B, Raghava J, Nordholm D, Randers L, et al. White matter maturation during 12 months in individuals at ultra-high-risk for psychosis. Acta Psychiatr Scandinavica. 2018;137:65-78.

12. Kristensen TD, Mandl RC, Raghava JM, Jessen K, Jepsen JRM, Fagerlund B, et al. Widespread higher fractional anisotropy associates to better cognitive functions in individuals at ultra-high risk for psychosis. Hum brain Mapp. 2019;40:5185-5201.

13. Tomyshev A, Lebedeva I, Akhadov T, Omel'chenko M, Ublinskii $\mathrm{M}$, Semenova N, et al. MRI study for the features of brain conduction pathways in patients with an ultra-high risk of endogenous psychoses. Bull Exp Biol Med. 2017;162:425-429.

14. Peters BD, de Haan L, Dekker N, Blaas J, Becker HE, Dingemans $\mathrm{PM}$, et al. White matter fibertracking in first-episode schizophrenia, schizoaffective patients and subjects at ultra-high risk of psychosis. Neuropsychobiology. 2008;58:19-28.

15. Peters BD, Dingemans PM, Dekker N, Blaas J, Akkerman E, van Amelsvoort TA, et al. White matter connectivity and psychosis in ultra-high-risk subjects: a diffusion tensor fiber tracking study. Psychiatry Res. 2010;181:44-50.

16. Schmidt A, Lenz C, Smieskova R, Harrisberger F, Walter A, Riecher-Rössler A, et al. Brain diffusion changes in emerging psychosis and the impact of state-dependent psychopathology. Neurosignals. 2015;23:71-83.

17. Fusar-Poli P, Cappucciati M, Borgwardt S, Woods SW, Addington J, Nelson B, et al. Heterogeneity of psychosis risk within individuals at clinical high risk: a meta-analytical stratification. JAMA psychiatry. 2016;73:113-120.

18. Chung Y, Cannon TD. Brain imaging during the transition from psychosis prodrome to schizophrenia. J Nerv Ment Dis. 2015;203:336-341.

19. Takahashi T, Wood SJ, Yung AR, Soulsby B, McGorry PD, Suzuki M, et al. Progressive gray matter reduction of the superior temporal gyrus during transition to psychosis. Arch Gen Psychiatry. 2009;66:366-376.

20. Cannon TD, Chung Y, He G, Sun D, Jacobson A, van Erp TGM, et al. Progressive reduction in cortical thickness as psychosis develops: a multisite longitudinal neuroimaging study of youth at elevated clinical risk. Biol psychiatry. 2015;77:147-157. 
21. Pantelis C, Velakoulis D, McGorry PD, Wood SJ, Suckling J, Phillips LJ, et al. Neuroanatomical abnormalities before and after onset of psychosis: a cross-sectional and longitudinal MRI comparison. Lancet. 2003;361:281-288.

22. Takahashi T, Wood SJ, Yung AR, Phillips LJ, Soulsby B, McGorry PD, et al. Insular cortex gray matter changes in individuals at ultra-high-risk of developing psychosis. Schizophrenia Res. 2009;111:94-102.

23. Ziermans TB, Schothorst PF, Schnack HG, Koolschijn PCM, Kahn RS, van Engeland $\mathrm{H}$, et al. Progressive structural brain changes during development of psychosis. Schizophrenia Bull. 2012;38:519-530.

24. Borgwardt SJ, McGuire PK, Aston J, Gschwandtner U, Pflüger MO, Stieglitz R-D, et al. Reductions in frontal, temporal and parietal volume associated with the onset of psychosis. Schizophrenia Res. 2008;106:108-114.

25. Walter A, Studerus E, Smieskova R, Kuster P, Aston J, Lang UE, et al. Hippocampal volume in subjects at high risk of psychosis: a longitudinal MRI study. Schizophrenia Res. 2012;142:217-222.

26. Sun D, Phillips L, Velakoulis D, Yung A, McGorry PD, Wood SJ, et al. Progressive brain structural changes mapped as psychosis develops in 'at risk'individuals. Schizophrenia Res. 2009;108:85-92.

27. Chung Y, Addington J, Bearden CE, Cadenhead K, Cornblatt B, Mathalon $\mathrm{DH}$, et al. Use of machine learning to determine deviance in neuroanatomical maturity associated with future psychosis in youths at clinically high risk. JAMA psychiatry. 2018;75:960-968.

28. Carletti F, Woolley JB, Bhattacharyya S, Perez-Iglesias R, Fusar Poli P, Valmaggia L, et al. Alterations in white matter evident before the onset of psychosis. Schizophrenia Bull. 2012;38:1170-1179.

29. Rigucci S, Santi G, Corigliano V, Imola A, Rossi-Espagnet C, Mancinelli I, et al. White matter microstructure in ultra-high risk and first episode schizophrenia: a prospective study. Psychiatry Res: Neuroimaging. 2016;247:42-48.

30. Peters BD, Szeszko PR, Radua J, Ikuta T, Gruner P, DeRosse P, et al. White matter development in adolescence: diffusion tensor imaging and meta-analytic results. Schizophrenia Bull. 2012;38:1308-1317.

31. Lebel C, Beaulieu C. Longitudinal development of human brain wiring continues from childhood into adulthood. J Neurosci. 2011;31:10937-10947.

32. Takahashi N, Sakurai T, Davis KL, Buxbaum JD. Linking oligodendrocyte and myelin dysfunction to neurocircuitry abnormalities in schizophrenia. Prog Neurobiol. 2011;93:13-24.

33. Roussos P, Haroutunian V Schizophrenia: susceptibility genes and oligodendroglial and myelin related abnormalities. Front Cell Neurosci. 2014;8:1-7.

34. Karayumak SC, Bouix S, Ning L, James A, Crow T, Shenton M, et al. Retrospective harmonization of multi-site diffusion MRI data acquired with different acquisition parameters. NeuroImage. 2019;184:180-200.

35. Cropley VL, Klauser P, Lenroot RK, Bruggemann J, Sundram S, Bousman C, et al. Accelerated gray and white matter deterioration with age in schizophrenia. Am J Psychiatry. 2016;174:286-295.

36. Di Biase MA, Cropley VL, Cocchi L, Fornito A, Calamante F, Ganella EP et al. Linking cortical and connectional pathology in schizophrenia. Schizophrenia Bull. 2018; sby 121.

37. Pasternak O, Sochen N, Gur Y, Intrator N, Assaf Y. Free water elimination and mapping from diffusion MRI. Magn Reson Med. 2009;62:717-730.

38. Addington J, Liu L, Brummitt K, Bearden CE, Cadenhead KS, Cornblatt BA et al. North American Prodrome Longitudinal Study (NAPLS 3): methods and baseline description. Schizophrenia Res. 2020. In Press.
39. McGlashan T, Walsh B, Woods S The psychosis-risk syndrome: handbook for diagnosis and follow-up. USA: Oxford University Press 2010.

40. Miller TJ, McGlashan TH, Rosen JL, Cadenhead K, Ventura J, McFarlane W, et al. Prodromal assessment with the structured interview for prodromal syndromes and the scale of prodromal symptoms: predictive validity, interrater reliability, and training to reliability. Schizophrenia Bull. 2003;29:703-715.

41. First MB, Gibbon M The Structured Clinical Interview for DSMIV Axis I Disorders (SCID-I) and the Structured Clinical Interview for DSM-IV Axis II Disorders (SCID-II). In: Hersen M, Haynes SN, Goldstein G, Heiby EM, Hilsenroth MJ, Beers SR et al. (eds). Comprehensive Handbook of Psychological Assessment, vol. 2: Personality Assessment. John Wiley \& Sons Inc: New Jersey, 2004, pp 134-143.

42. Ning L, Bonet-Carne E, Grussu F, Sepehrband F, Kaden E, Veraart J, et al. Cross-scanner and cross-protocol multi-shell diffusion MRI data harmonization: Algorithms and results. NeuroImage. 2020;221:117128.

43. Jenkinson M, Beckmann CF, Behrens TE, Woolrich MW, Smith SM. Fsl. NeuroImage. 2012;62:782-790.

44. Andersson JL, Sotiropoulos SN. An integrated approach to correction for off-resonance effects and subject movement in diffusion MR imaging. NeuroImage. 2016;125:1063-1078.

45. Andersson JL, Graham MS, Zsoldos E, Sotiropoulos SN. Incorporating outlier detection and replacement into a non-parametric framework for movement and distortion correction of diffusion MR images. NeuroImage. 2016;141:556-572.

46. Jahanshad N, Kochunov PV, Sprooten E, Mandl RC, Nichols TE, Almasy L, et al. Multi-site genetic analysis of diffusion images and voxelwise heritability analysis: a pilot project of the ENIGMA-DTI working group. NeuroImage. 2013;81:455-469.

47. Avants BB, Epstein CL, Grossman M, Gee JC. Symmetric diffeomorphic image registration with cross-correlation: evaluating automated labeling of elderly and neurodegenerative brain. Med image Anal. 2008;12:26-41.

48. Smith SM, Jenkinson M, Johansen-Berg H, Rueckert D, Nichols TE, Mackay CE, et al. Tract-based spatial statistics: voxelwise analysis of multi-subject diffusion data. NeuroImage. 2006;31:1487-1505.

49. Ilin A, Raiko T. Practical approaches to principal component analysis in the presence of missing values. J Mach Learn Res. 2010;11:1957-2000.

50. Winkler AM, Ridgway GR, Webster MA, Smith SM, Nichols TE. Permutation inference for the general linear model. NeuroImage. 2014;92:381-397.

51. Smith SM Nichols TE. Threshold-free cluster enhancement: addressing problems of smoothing, threshold dependence and localisation in cluster inference. NeuroImage. 2009;44: 83-98.

52. Smith SM, Nichols TE, Vidaurre D, Winkler AM, Behrens TE, Glasser MF, et al. A positive-negative mode of population covariation links brain connectivity, demographics and behavior. Nat Neurosci. 2015;18:1565-1567.

53. Taquet M, Smith SM, Prohl AK, Peters JM, Warfield SK, Scherrer B et al. A structural brain network of genetic vulnerability to psychiatric illness. Molecular Psychiatry. 2020: 1-12.

54. Dev SI, Nguyen TT, McKenna BS, Sutherland AN, Bartsch H, Theilmann RJ, et al. Steeper slope of age-related changes in white matter microstructure and processing speed in bipolar disorder. Am J Geriatr Psychiatry. 2017;25:744-752.

55. van Velzen LS, Kelly S, Isaev D, Aleman A, Aftanas LI, Bauer J, et al. White matter disturbances in major depressive disorder: a coordinated analysis across 20 international cohorts in the ENIGMA MDD working group. Mol Psychiatry. 2020;25: $1511-1525$. 
56. Hawco C, Voineskos AN, Radhu N, Rotenberg D, Ameis S, Backhouse FA, et al. Age and gender interactions in white matter of schizophrenia and obsessive compulsive disorder compared to non-psychiatric controls: commonalities across disorders. Brain imaging Behav. 2017;11:1836-1848.

57. Yeatman JD, Wandell BA, Mezer AA. Lifespan maturation and degeneration of human brain white matter. Nat Commun. 2014;5:4932-4932.

58. Miller EK, Cohen JD. An integrative theory of prefrontal cortex function. Annu Rev Neurosci. 2001;24:167-202.

59. Nakajima R, Kinoshita M, Shinohara H, Nakada M. The superior longitudinal fascicle: reconsidering the fronto-parietal neural network based on anatomy and function. Brain imaging Behav. 2020;14:2817-2830.

60. Barbu MC, Spiliopoulou A, Colombo M, McKeigue P, Clarke T$\mathrm{K}$, Howard DM, et al. Expression quantitative trait loci-derived scores and white matter microstructure in UK Biobank: a novel approach to integrating genetics and neuroimaging. Trans Psychiatry. 2020;10:55.

61. Tomassy GS, Dershowitz LB, Arlotta P. Diversity matters: a revised guide to myelination. Trends Cell Biol. 2016;26:135-147.

62. Di Biase MA, Zhang F, Lyall A, Kubicki M, Mandl RC, Sommer IE et al. Neuroimaging auditory verbal hallucinations in schizophrenia patient and healthy populations. Psychological Med. 2019: 1-10.
63. Tang Y, Pasternak O, Kubicki M, Rathi Y, Zhang T, Wang J, et al. Altered cellular white matter but not extracellular free water on diffusion MRI in individuals at clinical high risk for psychosis. Am J Psychiatry. 2019;176:820-828.

64. Becker MP, Collins PF, Lim KO, Muetzel RL, Luciana M. Longitudinal changes in white matter microstructure after heavy cannabis use. Dev Cogn Neurosci. 2015;16:23-35.

65. McGorry PD, Hartmann JA, Spooner R, Nelson B. Beyond the "at risk mental state" concept: transitioning to transdiagnostic psychiatry. World Psychiatry. 2018;17:133-142.

66. Woods SW, Powers AR III, Taylor JH, Davidson CA, Johannesen $\mathrm{JK}$, Addington $\mathrm{J}$, et al. Lack of diagnostic pluripotentiality in patients at clinical high risk for psychosis: specificity of comorbidity persistence and search for pluripotential subgroups. Schizophrenia Bull. 2018;44:254-263.

67. Fusar-Poli P, Rutigliano G, Stahl D, Davies C, De Micheli A, Ramella-Cravaro V, et al. Long-term validity of the at risk mental state (ARMS) for predicting psychotic and non-psychotic mental disorders. Eur Psychiatry. 2017;42:49-54.

68. Webb JR, Addington J, Perkins DO, Bearden CE, Cadenhead KS, Cannon TD, et al. Specificity of incident diagnostic outcomes in patients at clinical high risk for psychosis. Schizophrenia Bull. 2015;41:1066-1075.

\section{Affiliations}

Maria A. Di Biase $\mathbb{D}^{1,2} \cdot$ Suheyla Cetin-Karayumak $\mathbb{D}^{1,3} \cdot$ Amanda E. Lyall $\mathbb{D}^{1,4} \cdot$ Andrew Zalesky $^{2}$. Kang Ik Kevin $\mathrm{Cho}^{1} \cdot$ Fan Zhang $\mathbb{D}^{3} \cdot$ Marek Kubicki $\mathbb{D}^{1,3,4} \cdot$ Yogesh Rathi ${ }^{1,3} \cdot$ Monica G. Lyons ${ }^{1} \cdot$ Sylvain Bouix $\left(\mathbb{D}^{1}\right.$. Tashrif Billah $^{1}$ - Alan Anticevic ${ }^{5}$. Charlie Schleifer ${ }^{6}$ Brendan D. Adkinson ${ }^{7} \cdot$ Jie Lisa Ji $\oplus^{5} \cdot$ Zailyn Tamayo $^{5}$. Jean Addington $\mathbb{D}^{8} \cdot$ Carrie E. Bearden $\mathbb{D}^{9} \cdot$ Barbara A. Cornblatt ${ }^{10,11,12} \cdot$ Matcheri S. Keshavan $^{13}$. Daniel H. Mathalon ${ }^{14,15}$. Thomas H. McGlashan ${ }^{16}$. Diana O. Perkins ${ }^{11,17}$ - Kristen S. Cadenhead ${ }^{18}$. Ming T. Tsuang ${ }^{18} \cdot$ Scott W. Woods $s^{5}$. William S. Stone ${ }^{13} \cdot$ Martha E. Shenton $^{1,3,4} \cdot$ Tyrone D. Cannon $^{5}$. Ofer Pasternak $\mathbb{1 D}^{1,3}$

1 Department of Psychiatry, Brigham and Women's Hospital, Harvard Medical School, Boston, MA, USA

2 Melbourne Neuropsychiatry Centre, Department of Psychiatry, The University of Melbourne and Melbourne Health, Carlton South, VIC, Australia

3 Department of Radiology, Brigham and Women's Hospital, Harvard Medical School, Boston, MA, USA

4 Department of Psychiatry, Massachusetts General Hospital, Harvard Medical School, Boston, MA, USA

5 Department of Psychiatry and Psychology, Yale University, New Haven, CT, USA

6 UCLA School of Medicine, Los Angeles, CA, USA

7 Yale Interdepartmental Neuroscience Program, Yale School of Medicine, New Haven, CT, USA

8 Hotchkiss Brain Institute, University of Calgary, Calgary, AB, Canada

9 Department of Psychiatry and Biobehavioral Sciences, Semel Institute for Neuroscience and Human Behavior and Department of Psychology, University of California-Los Angeles, Los Angeles, CA, USA

10 Department of Psychiatry and Psychology, The Feinstein Institute for Medical Research, Manhasset, NY, USA

11 Department of Psychology, Hofstra North Shore-LIJ School of Medicine, Hempstead, NY, USA

12 The Zucker Hillside Hospital, New York, NY, USA

13 Harvard Medical School and Beth Israel Deaconess Medical Center, Boston, MA, USA

14 University of California, San Francisco, San Francisco, CA, USA

15 San Francisco VA Medical Center, San Francisco, CA, USA

16 Department of Psychiatry and Psychology, Yale University, New Haven, CT, USA

17 University of North Carolina (UNC), Chapel Hill, NC, USA

18 Department of Psychiatry, University of California San Diego (UCSD), La Jolla, CA, USA 